\title{
Comparison of the Static Balance Ability according to the Subjective Visual Vertical in Healthy Adults
}

\author{
Jung Won Kwon, Sang Seok Yeo \\ Department of Physical Therapy, College of Health Sciences, Dankook University, Cheonan, Korea
}

Purpose: The subjective visual vertical (SW) test is used to evaluate the otolith function in the inner ear. This study compared the different balance ability according to the results of the SW in healthy adults.

Methods: This study recruited 30 normal healthy subjects who did not have neurological and musculoskeletal disorders. The subjects were divided into experimental and control groups based on the results of SW: experimental group,$>2^{\circ}$; control group $<_{2} 2^{\circ}$. The static balance ability was evaluated using the Fourier Index, which could evaluate the balance capacity objectively and quantitatively.

Results: The mean angle of the SW in the experimental and control groups was $4.44^{\circ}$ and $0.59^{\circ}$, respectively. In the result of the Fourier series, the F1 frequency band in the experimental group showed a significantly higher value under one condition compared to the control group $(p<0.05)$. In the F2-4 and F5-6 frequency bands, the experimental group showed a significant increase in the Fourier series value under the four conditions compared to the control group $(p<0.05)$. In the F7-8 frequency band, significantly higher values of the Fourier series were observed in the experimental group under the three different conditions $(p<0.05)$.

Conclusion: These results showed increased trunk sway while maintaining static balance in the experimental group who showed a larger SW angle compared to the control group. The SW can be applied to evaluate the vestibular system and balance ability in normal adults.

Keywords: Subjective visual vertical, Static balance, Vision, Vestibular, Somatosensory

\section{INTRODUCTION}

Balance is an essential element in performing the functional activity based on the ability to maintain the center of the gravity on the base of support. ${ }^{1-4}$ The balance of the human body is maintained based on the vestibular, somatosensory, and visual information integrated into the central nervous system. ${ }^{5}$ Each sensory information for balance is mobilized in different priorities according to the different balance-maintenance situations. When problems occur in the transmission or interpretation of sensory information, this information also affects the maintenance of balance ability. ${ }^{6}$ The loss of balance ability interferes with the functional activities of everyday life and further leads to secondary problems, such as falls or fractures?

The vestibular system plays a major role in posture control and spatial perception by detecting the acceleration, tilt, and gravity of

Received May 13, 2020 Revised Jun 10, 2020

Accepted Jun 18, 2020

Corresponding author Sang Seok Yeo

E-mail yeopt@dankook.ac.kr the head. ${ }^{4,7}$ Unlike other sensory systems, the changes in the vestibular functions are relatively difficult to measure. ${ }^{1,2,89}$ Sensitive measurement techniques have been required to evaluate the central and peripheral vestibular system function in patients with neurological disorders. ${ }^{5,10}$ On the other hand, many studies reported that an evaluation of the balance function and visual system could be used to evaluate the vestibular function, even when a direct evaluation of the vestibular system was not included. ${ }^{11-14}$

The subjective visual vertical (SVV) is a tool for evaluating the function of the utricle in the inner ear and aims to assess the perception of verticality or abnormally tilted visual sensation. The SVV compares the subjective and actual vertical lines felt by the test subject, and in healthy adults, differences may occur in the range of approximately $2^{0.15,16}$ On the other hand, a difference in tilt of more than $4^{\circ}$ based on the standard deviation indicates a problem with the SVV line with respect to the actual gravity vertical line..$^{15,16}$ Sen-

Copylight (C)2020 The Korean Society of Physical Therapy

This is an Open Access article distribute under the terms of the Creative Commons Attribution Non-commercial License (https:// creativecommons.org/license/by-nc/4.o.) which permits unrestricted non-commercial use, distribution, and reproduction in any medium, provided the original work is properly cited. 
sory information received from the vestibular system is involved in the movement of the ocular muscles and control of the position of the eye via the medial longitudinal fasciculus, the oculomotor nerve, trochlear nerve, and abducens nerve..$^{17-19}$ Therefore, the vestibular system can be evaluated indirectly by observing the eye movement or perception of spatial information. ${ }^{7,12-16}$

Many previous studies reported the increasing pattern of SVV and the resulting difference in balance and walking ability in patients with peripherial or central vestibular disorders. ${ }^{9,14,20-23}$ However, there was no study which reported the effect of increased SVV titl in normal adults on static balance. Therefore, this study examined the difference in static balance function according to the SVV results in healthy young adults.

\section{METHODS}

\section{Subjects}

The SVV test was performed on 30 healthy young adults who had no neurological and musculoskeletal disorders related to balance ability and had no problems with daily life. The inclusion criteria were as follows: 1) no history of musculoskeletal, neurological, and cognitive problems, 2) independently performing the activities of daily living (ADL) and walking, 3) no direct problems with the vestibular system. The experimental group included 15 subjects who showed a significant increase in the SVV result, i.e., more than two standard deviations in reference to a prior study $(1.51 \pm 0.69) .{ }^{24}$ The control group included 15 subjects who showed the lowest displacement value among the subjects who showed $\leq 2^{\circ}$ in the SVV test. All participants provided informed consent, and the study was approved by the institutional review board at Dankook University.

\section{Measurements}

\section{1) Subjective Visual Vertical (SVV)}

A plastic cylindrical bucket was used to measure the SVV. The bucket was made of inexpensive and easily obtainable materials. A vertical straight line was drawn inside its bottom, and a protractor was attached outside its bottom to align with the protractor's vertical and zero lines. ${ }^{25,26}$ A pendulum was hung outside the bucket to match the protractor's zero line. ${ }^{25,26}$ The bucket was rotated randomly and given to the participants. The subjects were then asked to look at the line inside the bucket and to align the line vertically.
When they thought that this had occurred, they told the experimenters who documented the angles deviating from the zero line. The angles were recorded as positive regardless of the clockwise or counterclockwise directions. The SVV measurements were taken in a dark place to avoid a reflection of the protractor. The SVV results were averaged after three repetitions.

\section{2) Static balance test}

Static balance tests were evaluated using Tetrax (Sunlight Medical Ltd., Ramat Gan, Israel). The subjects placed their toes and heels on four platforms (A: left heel, B: left toe, C: right heel, D: right toe) and maintained a comfortable posture. The static balance test was performed in the following eight posture conditions, and the test time in each posture was 32 seconds.

NO: Neutral head position, eyes open, firm surface

NC: Neutral head position, eyes closed, firm surface

PO: Neutral head position, eyes open, elastic surface

PC: Neutral head position, eyes closed, elastic surface

HR: Head turned right about $45^{\circ}$, eyes closed, firm surface

HL: Head turned left about $45^{\circ}$, eyes closed, firm surface

HB: Head raised backward about $30^{\circ}$, eyes closed, firm surface

HF: Head bent forward about $30^{\circ}$, eyes closed, firm surface

The Fourier Index was measured during the balance test for each posture. The Fourier Index is classified into four frequency bands as an analysis method for postural sway on a scale of the rising frequency band. ${ }^{23-25} \mathrm{~F} 1$ (a low frequency of 0.01 to $0.1 \mathrm{~Hz}$ ) is associated with a visual system for balance control. The F2-F4 band (low to medium frequency of 0.1 to $0.5 \mathrm{~Hz}$ ) is associated with the peripheral vestibular system and can be increased by vestibular impairment or muscle fatigue. The F5-F6 band (medium-high frequency between 0.5 and 1.0 $\mathrm{Hz}$ ) is associated with the somatosensory system in the lower extremities, spine, and lower back. The high-frequency F7-F8 band (1.0 $\mathrm{Hz}$ or higher frequencies) is caused by a dysfunction of the central nervous system. ${ }^{27}$

\section{Statistical Analysis}

Data analysis was performed using SPSS 20.0 software (SPSS, Chicago, IL, USA). An independent t-test was used to compare the difference in the demographic data and each parameter between the experimental and control groups. Statistical significance was set to 0.05 . 


\section{RESULTS}

Table 1 summarizes the demographic data. The SVV results showed a significant increase in the experimental group compared to the control group $(\mathrm{p}<0.05)$.

As a result of the F1 comparison, the F1 frequency band showed a significantly higher frequency during the HR condition in the experimental group compared to the control group $(\mathrm{p}<0.05)$, but there was no significant difference in other conditions between the two groups ( $p>0.05$ ). In the F2-4 frequency band, the PC, HR, HL, and HF conditions showed significantly higher frequencies in the

Table 1. Demographic data of the experimental group and control group

\begin{tabular}{lcc}
\hline & $\begin{array}{c}\text { Experimental group } \\
(n=15)\end{array}$ & $\begin{array}{c}\text { Control group } \\
(n=15)\end{array}$ \\
\hline Age (yr) & $20.44 \pm 1.98$ & $20.69 \pm 1.76$ \\
Gender (Male/Female) & $6 / 9$ & $8 / 7$ \\
SW (deg) & & \\
\hline
\end{tabular}

Values represent mean ( \pm standard deviation), SW: subjective visual vertical, Independent t-test was used to compare the difference of demographic data between the experimental group and control group.

${ }^{*} \mathrm{p}<0.05$. experimental group than the control group $(\mathrm{p}<0.05)$. In the F5-6 frequency band, the NO, HR, HL, and HF conditions showed a significantly higher frequency in the experimental group than the control group ( $\mathrm{p}<0.05)$. In the F7-8 frequency band, the HR, HL, and HF conditions showed a significantly higher frequency in the experimental group than the control group $(\mathrm{p}<0.05)$. On the other hand, there were no significant differences between the two groups under the other conditions $(\mathrm{p}>0.05)$ (Table 2).

\section{DISCUSSION}

This study examined the difference in static balance ability according to the results of the SVV in healthy young adults. Based on the results of the SVV test, the subjects were classified into an experimental group and a control group; the experimental and control groups showed an average of $4.44^{\circ}$ and $0.59^{\circ}$, respectively. The results of the static balance test between the experimental and control groups are as follows. 1) In the F1 frequency band, only the HR condition showed a significant difference between the experimental and control groups. 2) In the F2-4 frequency band, there were significant differences in the PC, HR, HL, and HF conditions. 3) In the

Table 2. Comparison of four conditions for fourier series between experimental group and control group

\begin{tabular}{|c|c|c|c|c|c|c|}
\hline \multirow{2}{*}{ Condition } & \multicolumn{3}{|c|}{ F1 $(0.01 \sim 0.1 \mathrm{~Hz})$} & \multicolumn{3}{|c|}{$\mathrm{F} 2-4(0.1$ to $0.5 \mathrm{~Hz})$} \\
\hline & EG & CG & $\mathrm{p}$ & EG & CG & $p$ \\
\hline NO & 18.55 & 18.04 & 0.935 & 8.30 & 7.08 & 0.186 \\
\hline NC & 15.71 & 15.40 & 0.869 & 8.87 & 7.11 & 0.102 \\
\hline $\mathrm{PO}$ & 25.32 & 25.62 & 0.958 & 9.63 & 8.15 & 0.253 \\
\hline PC & 31.22 & 23.89 & 0.161 & 11.67 & 9.29 & $0.035^{*}$ \\
\hline$H R$ & 14.38 & 9.40 & $0.023^{*}$ & 8.59 & 5.87 & $0.001^{*}$ \\
\hline $\mathrm{HL}$ & 14.95 & 12.24 & 0.260 & 8.30 & 6.52 & $0.026^{*}$ \\
\hline $\mathrm{HB}$ & 19.23 & 19.70 & 0.933 & 9.35 & 7.89 & 0.23 \\
\hline \multirow[t]{3}{*}{$\mathrm{HF}$} & 15.54 & 13.47 & 0.461 & 9.49 & 7.68 & $0.048^{*}$ \\
\hline & \multicolumn{3}{|c|}{ F5-6 $(0.5$ and $1.0 \mathrm{~Hz})$} & \multicolumn{3}{|c|}{$\mathrm{F} 7-8(\geq 1.0 \mathrm{~Hz})$} \\
\hline & EG & CG & $p$ & EG & CG & $\mathrm{p}$ \\
\hline NO & 3.10 & 2.42 & $0.033^{*}$ & 0.63 & 0.51 & 0.099 \\
\hline NC & 3.23 & 2.59 & 0.121 & 0.62 & 0.48 & 0.112 \\
\hline $\mathrm{PO}$ & 3.92 & 3.34 & 0.126 & 0.71 & 0.63 & 0.36 \\
\hline PC & 4.52 & 3.99 & 0.349 & 0.91 & 0.69 & 0.096 \\
\hline $\mathrm{HR}$ & 3.56 & 2.35 & $0.003^{*}$ & 0.64 & 0.4 & $0.001^{*}$ \\
\hline $\mathrm{HL}$ & 3.87 & 2.42 & $0.008^{*}$ & 0.68 & 0.42 & $0.002^{*}$ \\
\hline $\mathrm{HB}$ & 3.46 & 3.09 & 0.479 & 0.70 & 0.59 & 0.29 \\
\hline $\mathrm{HF}$ & 4.00 & 2.58 & $0.003^{*}$ & 0.73 & 0.48 & $0.023^{*}$ \\
\hline
\end{tabular}

NO: eye open, NC: eye closed, PO: foam-rubber pillow with eye open, PC: foam-rubber pillow with eye closed, HR: head turned right and eyes closed, HL: head turned left and eyes closed, HB: head raised backward and eyes closed, HF: head bended forward and eyes closed, EG: experimental group: CG: control group.

${ }^{*} p<0.05$ 
F5-6 frequency band, there were significant differences in the NO, HR, HL, and HF conditions. 4) In the F7-8 frequency band, significant differences were noted in the HR, HL, and HF conditions.

The F1 frequency band corresponds to an evaluation of the visual system among sensory functions related to the balance ability, the F2-4, F5-6, and F7-8 frequency band corresponds to the peripheral vestibular system, somatosensory system, and central nervous system, respectively. ${ }^{27}$ Each frequency band evaluates the postural fluctuations that occur while maintaining a static balance, and frequencies F1 to F8 represent low to high frequencies. As a result, if the SVV result exceeds the normal range, it is believed that a change in static balance ability occurs even in young adults. In particular, in all four frequency bands, the postural fluctuation was increased when the head position was changed while the visual information was blocked. Therefore, an increase in SVV value can affect the integration of the somatosensory, peripheral, and central vestibular systems related to the posture verticality.

Many studies have reported that an increased SVV in patients with injury of the central or peripheral vestibular system can be related to the balance dysfunction. ${ }^{16,22,23,28}$ Pereira et al..$^{22}$ reported a correlation between the SVV and postural balance in Parkinson's disease patients. They suggested that patients with an impaired balance ability showed a large deviation of the SVV. In contrast, patients with good balance ability reported a small deviation of the SVV. ${ }^{22}$ Kim et al. ${ }^{23}$ reported two patients who showed isolated body lateropulsion due to a lateral medullary infarction. Both patients were associated with impaired subjective vertical values. ${ }^{23}$ Toupet et al. ${ }^{28}$ compared the SVV differences in patients with various vestibular dysfunctions according to age and sex. They suggested that female patients with a vestibular disorder showed a higher visual attraction index in all age groups. In particular, the effects of sex on the visual attraction index had the greatest effect on patients with benign paroxysmal postural vertigo. ${ }^{28}$ Cho et al. ${ }^{16}$ reported on the different gait characteristics between subjects with an increased SVV and subjects with a normal range SVV. The subjects with the increased SVV showed significantly higher stride time variability and stride velocity variability than the control group. Consequently, the SVV test can be used to assess the balance ability and perception of vertical posture according to the central vestibular disorder as well as the peripheral vestibular function related to the otolithic organs. In addition, the change in SVV can be related to the change in posture control function because of a central or peripheral vestibular disorder.

In conclusion, there were significant differences in the static balance ability under various conditions between the experimental and control groups. In addition, a higher SVV in young adults can influence the static balance under conditions, such as visual block or change of posture. These study findings on the static balance will help identify intervention strategies to treat patients with a risk of falls due to an injury of the vestibular system. On the other hand, the limitations of this study should be considered. First, the subjects were healthy young adults, which made it difficult to generalize the results to other age groups. Second, the SVV test does not use a quantified machine. Hence, an error can occur due to insufficient exercise ability to reflect the individual's sensory ability sufficiently because the subject adjusts the angle by directly moving the tool. Therefore, future studies with more subjects and objective and quantitative measurement methods will be needed.

\section{REFERENCES}

1. Horak FB, Wrisley DM, Frank J. The balance evaluation systems test (bestest) to differentiate balance deficits. Phys Ther. 2009;89(5):484-98.

2. Chandler JM, Duncan PW, Studenski SA. Balance performance on the postural stress test: Comparison of young adults, healthy elderly, and fallers. Phys Ther. 1990;70(7):410-5.

3. Nichols DS, Glenn TM, Hutchinson KJ. Changes in the mean center of balance during balance testing in young adults. Phys Ther.1995;75(8):699706.

4. Wade MG, Jones G. The role of vision and spatial orientation in the maintenance of posture. Phys Ther. 1997;77(6):619-28.

5. Lee HJ, Song CH, Lee KJ et al. The effects of complex exercise training for lower legs muscle strength, muscle endurance, balance ability and gait ability in the elderly. Journal of Sport and Leisure Studies. 2010;41(2):935-47.

6. Park KS. Principles of sensory integration and teaching techniques. Seoul, A Publishing Special Education, 1991.

7. Zalewski CK. Aging of the human vestibular system. Semin Hear. 2015;36(3):175-96.

8. Kim CR, Chun MH, Lee GA. Assessments of balance control using tetraataxiametric posturography. Ann Rehabil Med. 2009;33(4):429-35.

9. Cronin T, Arshad Q, Seemungal BM. Vestibular deficits in neurodegenerative disorders: balance, dizziness, and spatial disorientation. Front Neurol. 2017;8:538.

10. Uloziene I, Totiliene M, Paulauskas A et al. Subjective visual vertical assessment with mobile virtual reality system. Medicina (Kaunas).2017; 53(6):394-402.

11. Ramat S. Chapter 3 - understanding the rotational vestibular ocular reflex: from differential equations to laplace transforms. In: Ramat S, Shai- 
kh AG, eds, Progress in brain research, Elsevier, 2019:29-44.

12. Honaker JA, Janky KL, Patterson JN et al. Modified head shake sensory organization test: sensitivity and specificity. Gait Posture. 2016;49:67-72.

13. Heick JD, Bay C, Dompier TP et al. Relationships among common vision and vestibular tests in healthy recreational athletes. Int J Sports Phys Ther. 2017;12(4):581-91.

14. Ashish G, Augustine AM, Tyagi AK et al. Subjective visual vertical and horizontal in vestibular migraine. J Int Adv Otol. 2017;13(2):254-8.

15. Akin FW, Murnane OD, Pearson A et al. Normative data for the subjective visual vertical test during centrifugation. J Am Acad Audiol. 2011;22(7):460-8.

16. Cho IH, Kwon JW, Yeo SS. Relations between gait characteristics and subjective visual vertical results in young adults. J Vestib Res. 2020; 6(10):VES-200694.

17. Sakai K, Yokota H, Akazawa K et al. Brainstem white matter tracts and the control of eye movements. Semin Ultrasound CT MR. 2014;35(5):517-26.

18. Sciacca S, Lynch J, Davagnanam I et al. Midbrain, pons, and medulla: anatomy and syndromes. Radiographics. 2019;39(4):1110-25.

19. McNulty JP, Lonergan R, Bannigan J et al. Visualisation of the medial longitudinal fasciculus using fibre tractography in multiple sclerosis patients with internuclear ophthalmoplegia. Ir J Med Sci. 2016;185(2):393402.

20. Quitschal RM, Fukunaga JY, Gananca MM et al. Evaluation of postural control in unilateral vestibular hypofunction. Braz J Otorhinolaryngol.
2014;80(4):339-45.

21. Aydin E, Metin Tellioglu A, Kurt Omurlu I et al. Postural balance control in women with generalized joint laxity. Turk J Phys Med Rehabil. 2017;63(3):259-65.

22. Pereira CB, Kanashiro AK, Maia FM et al. Correlation of impaired subjective visual vertical and postural instability in parkinson's disease. J Neurol Sci. 2014;346(1-2):60-5.

23. Kim JH, Kim S, Lee DH et al. Isolated axial lateropulsion with ipsilesional subjective visual vertical tilt in caudal lateral medullary infarction. J Vestib Res. 2015;25(1):41-5.

24. Ashish G, Augustine A, Kumar Tyagi A et al. Subjective visual vertical and horizontal: normative values using a software-based test in the indian population. Indian Journal of Otology. 2016;22:208.

25. Zwergal A, Rettinger N, Frenzel C et al. A bucket of static vestibular function. Neurology. 2009;72(19):1689-92.

26. Ferreira MM, Cunha F, Gananca CF et al. Subjective visual vertical with the bucket method in brazilian healthy individuals. Braz J Otorhinolaryngol. 2016;82(4):442-6.

27. Choi BR. The effects of hippotherapy on standing balance in patients with incomplete cervical spinal cord injuries: a pilot study. Neuroscience \& Medicine. 2013;04:7-15.

28. Toupet M, Van Nechel C, Grayeli AB. Subjective visual vertical tilt attraction to the side of rod presentation: effects of age, sex, and vestibular disorders. Otol Neurotol. 2015;36(6):1074-80. 